\title{
Rapid Detection of Methecillin Resistant Staphylococcus Aureus Using Loop Mediated Isothermal Amplification (LAMP)
}

\section{Alaa Fathelrahman ${ }^{1}$, Abdel Rahim M El Hussein ${ }^{2}$, Abd Elrahman Hassan Ishag ${ }^{1}$, Hassan Madni' ${ }^{2}$, Mohamed 0 Mustafa' ${ }^{2}$, Razan A Bashir ${ }^{2}$, Tarteel Hassan ${ }^{2}$, Isam M Elkhidir ${ }^{3}$ and Khalid A Enan ${ }^{2 *}$}

${ }^{1}$ Ahfad University for Women, Sudan

${ }^{2}$ Central Laboratory, Ministry of High Education and Scientific Research, Khartoum, Sudan

${ }^{3}$ Department of Microbiology and Parasitology, Faculty of Medicine, University of Khartoum, Khartoum, Sudan

*Corresponding Author: Khalid A Enan, Department of Microbiology and Parasitology, Faculty of Medicine, University of Khartoum, Khartoum, Sudan.

Received: September 26, 2019; Published: October 22, 2019

DOI: $10.31080 /$ ASMI.2019.02.0408

\begin{abstract}
Background: Staphylococcus aureus, including methicillin-resistant S. aureus (MRSA), is a major bacterial pathogen associated with nosocomial and community-acquired $S$. aureus infections all over the world.

Aim: The aim of this study was to establish the loop mediated isothermal amplification technique as a rapid detection method for methicillin resistant $\mathrm{S}$. aureus.

Methods: The study was carried out in Omdurman Military hospital, Alshorta hospital, and Alneelain university dental clinic. A total of 60 samples were collected and cultured, All confirmed $S$. aureus isolates were tested against oxacillin antibiotic by the disk diffusion method to detect MRSA isolates LAMP and PCR assays were then used to detect mec A gene directly from the samples and from culture isolates.

Results: Among 60 (wound, dental plaque and urine) seven samples (11.6\%) MRSA were detected by culture, 5\% were found positive for mec A using LAMP and 3.3\% using PCR. LAMP detected mec A in 100\% of the seven MRSA isolates, PCR detected mec A in $14.3 \%$. The LAMP results were confirmed using melting analysis.

Conclusion: The LAMP described herein is a reliable and rapid method for detection of the mec A gene. Generally, these findings are useful for future studies since there is little available information about MRSA infection in Sudan. LAMP can be used in a hospital setting for rapid diagnosis of MSRA, this should help better management and treatment of the affected patients in addition to rapid initiation of infection control procedures.
\end{abstract}

Keywords: S. aureus; mec A; LAMP; PCR

\section{Abbreviations}

PCR: Polymerase Chain Reaction; LAMP: Loop Mediated Isothermal Amplification; MRSA: Methicillin Resistant Staphylococcus aureus; BST: Bacillus stearothermophilus; SCCMEC: Staphylococcal Chromosomal Cassette mec mobile genetic element; UV: Ultra Violet; DNA: Deoxyribonucleic Acid

\section{Introduction}

Staphylococcus aureus is an infectious pathogen that can survive on inanimate environmental surfaces. It can colonize skin, mucous membranes, and the anterior nares in about $30 \%$ of healthy individuals [1]. They grow well under conditions of high osmotic pressure and low moisture, making them able to grow and survive in 
nasal secretions and on the skin [2]. Staphylococcus aureus is found in a wide range of habitats including environmental surfaces, in nasal nares of domesticated animals like dogs, cats and horses and on human body surfaces as part of normal microflora [3]. The human body is a natural reservoir for this bacterium, and studies have shown that the anterior nares are where it is often found than in the other parts of the body [4]. Carriers may be divided into three groups: persistent, intermittent, and non-carriers Persistent carriers usually carry only one strain and make up $20 \%$ of the population. Sixty percent of intermittent carriers harbor multiple strains of S. aureus for weeks at a time. Twenty percent are non-carriers and may yield negative results in the laboratory cultures on repeat swabs over time [5]. Methicillin-resistant Staphylococcus aureus (MRSA) is associated with substantial morbidity and mortality in many regions of the world [1]. Methicillin-susceptible S. aureus becomes methicillin-resistant $S$. aureus (MRSA) upon acquisition of the Staphylococcal Chromosomal Cassette mec (SCC mec) mobile genetic element. SCC mec harbors mec A or mec C, both of which encode alternate penicillin- binding proteins, which mediate resistance to almost all $\beta$-lactam antibiotics [6]. Rapid identification of MRSA in hospitalized patients is essential for administration of appropriate antibiotic therapy and infection control regimens. In addition, the increasing numbers of community-acquired infections necessitate the screening of carriers, rather than simply detecting the infected patient [7]. Staphylococcus strains have commonly been identified via routine standard procedures including colony morphology, Gram staining, testing of catalase, and coagulase [8], MRSA is identified via susceptibility tests [9], The disadvantage of the phenotypic methods are that they are time consuming and have a turnaround time of 2-3 days [10].

Identification of MRSA by molecular methods is widely used and based; in general; on the detection of $S$. aureus specific gene target the mec A gene [11]. For the rapid examination of methicillin resistance in Staphylococci, polymerase chain reaction (PCR)based molecular techniques have been developed by targeting the mecA, the gene for penicillin-binding protein $2 \mathrm{a}$ [12], and real-time PCR is now widely used for the diagnosis [13]. In general, compared to the conventional culture method, PCR can be performed in relatively rapid and simple fashion, but special reagents and apparatus such as thermal cycler are needed for the method. In the last decade, loop-mediated isothermal amplification (LAMP) had been reported as a novel nucleic acid amplification method and ap- plied to the detection of various pathogenic organisms [8]. LAMP which is based on auto cycling strand displacement DNA synthesis using the Bst DNA polymerase enzyme was developed by Notomi., et al [14]. The Bst DNA polymerase was isolated from Bacillus stearothermophilus, which possesses a $5^{\prime} \rightarrow 3^{\prime}$ exonuclease along with $5^{\prime} \rightarrow 3^{\prime}$ polymerase activity and needs high concentration of magnesium for optimum activity [15]. The LAMP reaction can be conducted under isothermal condition ranging from 60 to 65 C [16,17], and specificity is attributable to four primers that recognize six distinct sequences [14-18]. Continuous amplification under isothermal condition produces an extremely large amount of target DNA within less than an hour $[7,19]$, and the method enables simple visual (naked-eye) judgment of the DNA amplification through a white precipitate [20] or color change of the reaction mixture with SYBR green I [21]. A number of LAMP tests to detect MRSA have been designed and used successfully [8,22]. The rapidity, specificity, and simplicity of the technique make it appealing for use in MRSA screening. The purpose of the present study was establishment of a LAMP test for detection of MRSA based on the mecA gene and to compare it with a PCR method.

\section{Material and methodology}

Study design

This is a Cross sectional study carried out in Khartoum State's hospitals.

\section{Clinical samples}

60 miscellaneous samples were kindly provided by the following:

Omdurman military hospital (20 wound samples), Alshorta hospital (20 urine samples)., Alneelain university dental clinic (20 dental plaque samples). The patients did not have a medical history of MRSA-infection.

Isolation, identification, and antimicrobial susceptibility testing

The samples were cultured using standard methods on mannitol salt agar at $37^{\circ} \mathrm{C}$ for 24 hours, Isolation of $S$. aureus was based on the morphology of colonies, mannitol fermentation ability, catalase and coagulase tests. All confirmed $S$. aureus isolates were tested against oxacillin antibiotic by the disk diffusion method to detect MRSA isolates. 


\section{DNA Extraction}

DNA was extracted from positive-MRSA isolates, directly from the samples using boiling method.

PCR

For PCR, backward and forward primers mentioned in (Table 1) were used to amplify mecA region, The reaction was performed in $20 \mu$ volume using Solis Bio dyne master mix.( Solis Bio dyne, Estonia ) The volume included: $4 \mu \mathrm{l}$ master mix, $1 \mu \mathrm{l}$ forward primer, $1 \mu \mathrm{l}$ reverse primer, $5 \mu \mathrm{l}$ extracted DNA and $9 \mu \mathrm{l}$ distilled water. The DNA was amplified in thermocycling conditions using PCR machine Techno (Japan) as follows: initial denaturation at $94^{\circ} \mathrm{C}$ for $5 \mathrm{~min}$, followed by 35 cycles of denaturation at $94^{\circ} \mathrm{C}$ for $1 \mathrm{~min}$, annealing at $62^{\circ} \mathrm{C}$ for $1 \mathrm{~min}$ and extension at $72^{\circ} \mathrm{C}$ for $1 \mathrm{~min}$, with a final extension at $72^{\circ} \mathrm{C}$ for $7 \mathrm{~min}$. Each amplicon was run in $2 \%$ agarose gel. MRSA clinical samples and culture isolates that were successfully amplified gave bands of $210 \mathrm{bp}$ when the gel was visualized under UV light, this was considered as positive result for mecA gene presence.

\begin{tabular}{|l|c|}
\hline Primers & Sequence \\
\hline Forward & 5'-TGCTATCCACCCTCAAACA GG-3' $^{\prime}$ \\
\hline Reverse & 5'-AACGTTGTAACCACCCCAAGA-3' \\
\hline
\end{tabular}

Table 1: PCRuniversal primers sequence used for the amplification of mecA gene [7].

\section{LAMP primers Mix}

Primers mix that contains four primers (FIP, BIP, F3, B3) which were derived from the sequence of the mecA gene [7] were used. Final concentration of FIP and BIP was 40 pmol each, F3 and B3 5 pmol each. The sequences of these primers are shown in (Table 2).

\begin{tabular}{|l|c|}
\hline Primer & Sequence \\
\hline F3 & $5^{\prime}$-aagatggcaaagatattcaact-3' \\
B3 & $5^{\prime}$-aggttcttttttatcttcggtta-3' \\
FIB & $5^{\prime}$-acctgtttgagggtggatagcatgatgctaaagttcaaaagagt-3' \\
BIP & $5^{\prime}$-gcacttgtaagcacaccttcacttcgttactcatgccatac-3' \\
\hline
\end{tabular}

Table 2: LAMP Primers used in this study [7].

\section{LAMP condition}

The reaction was performed in a final volume of $25 \mu \mathrm{l}$ using LAMP regents (Mast, Reinfeld, Germany) which contained $12.5 \mu \mathrm{l}$ $2 x$ LAMP reaction buffer, $1 \mu$ of Bst DNA polymerase, $2 \mu$ primer mix (PM) included (40 pmol each FIP and BIP primers, 5 pmol each F3 and B3 primers), $1 \mu$ l fluorescence dye (FD), $6.5 \mu \mathrm{l} \mathrm{H} 20$ and 2 $\mu \mathrm{l}$ of target DNA. The mixture was incubated in a real-time PCR at $64 \mathrm{o} C$ for 60 minutes and the results were visualized using the FAM channel.

\section{Result}

Antimicrobial Susceptibilities Test

Among a total of 60 samples 7(11.6\%) MRSA were isolated.

\section{PCR detection of mecA gene among MRSA isolates and clinical} samples

PCR amplification showed that two out of sixty (3.3\%) samples were positive for mecA gene for the directly extracted samples, and one out of seven(14.3\%) samples was positive for mecA gene for the isolated MRSA.

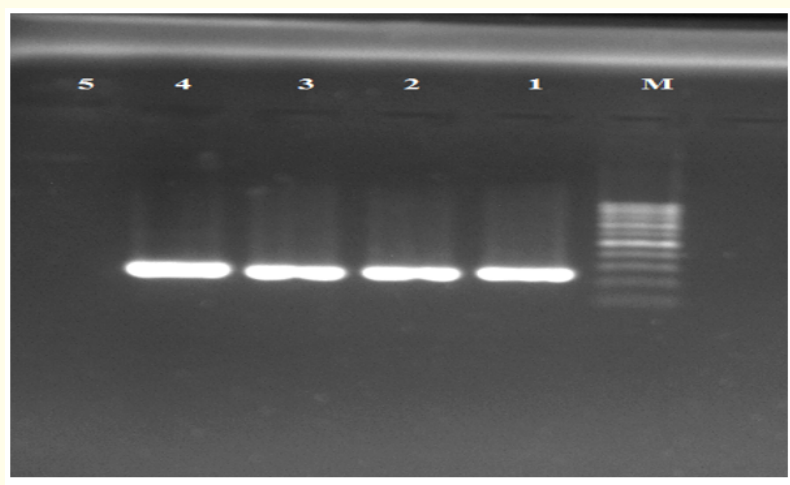

Figure 1: MecA PCR result (210bp) on 2\% agrose gel. Lane1and 2shows positive control, lanes, 3 and 4 show results in two patients, lane 5 show negative control, M: $100 \mathrm{bp}$ DNA marker.

LAMP detection of mecA gene among MRSA isolates and clinical samples

LAMP amplification showed that three out of sixty (5\%) samples were positive for mecA gene for the directly extracted samples, and seven out of seven (100\%) samples was positive for mecA gene for the isolated MRSA. 


\begin{tabular}{|l|c|c|c|c|c|}
\hline \multirow{2}{*}{} & \multicolumn{3}{|c|}{ Culture Samples } & \multicolumn{2}{c|}{ Clinical Samples } \\
\cline { 2 - 6 } & Culture & LAMP & PCR & LAMP & PCR \\
\hline Positive & $7(11.6 \%)$ & $7(100 \%)$ & $1(14.3 \%)$ & $3(5 \%)$ & $2(3.3 \%)$ \\
\hline Negative & $53(88.4 \%)$ & $0(0 \%)$ & $6(85.7 \%)$ & $57(95 \%)$ & $58(96.7 \%)$ \\
\hline Total & $60(100 \%)$ & $7(100 \%)$ & $7(100 \%)$ & $60(100 \%)$ & $60(100 \%)$ \\
\hline
\end{tabular}

Table3: Comparison of the results for mecA LAMP and mecA PCR using clinical samples and MRSA isolates.

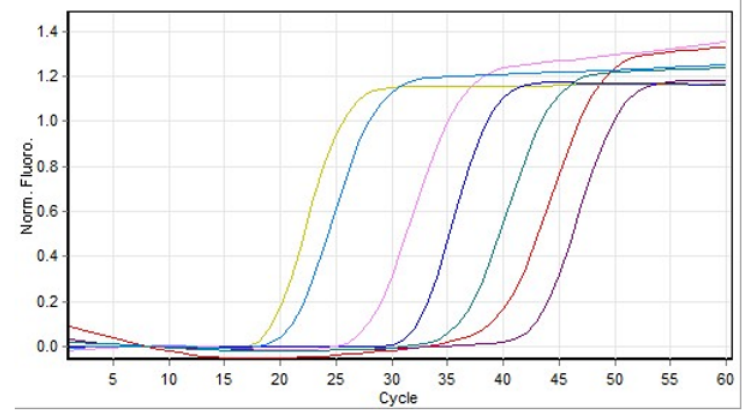

A

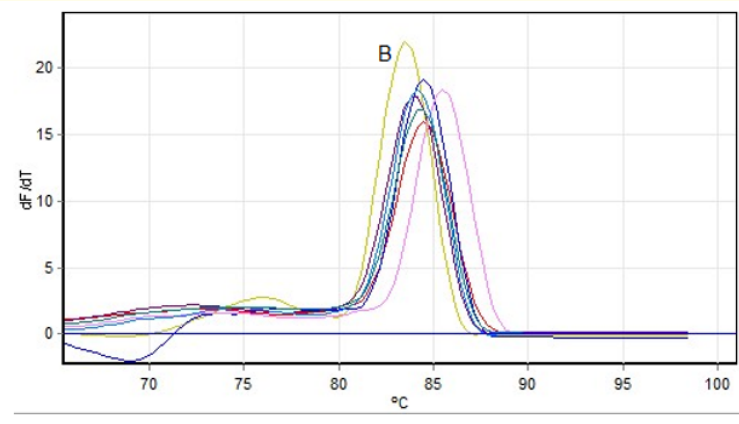

B

Figure 2: Examples for Amplification plot and melt curve of LAMP. A. amplification analysis, B. melting-curve analysis. Beak B in yellow color indicate the positive control.

\section{Discussion}

In the current study, LAMP assay was used for the rapid identification of MRSA from pure cultures and directly from wound, dental plaque, and urine samples using simple boiling DNA extraction method. A rapid and accurate LAMP assay for detection of MRSA is described. Compared with conventional PCR, which consumes four hours until reading the result under UV, LAMP reactions are characteristically rapid and are performed to higher amplification efficiency within sixty minutes. This is due to the isothermal nature of LAMP, as it proceeds at the optimum temperature of the polymerase, with no loss of time resulting from changes in the temperature of the reaction system [23].

LAMP detected mecA gene in all seven MRSA culture isolates while PCR detected mecA gene in only one sample which is similar to the results of $[24,25]$ who reported that LAMP is 10 times more sensitive than standard PCR. The lesser sensitivity of PCR than the LAMP method may be due to the presence of PCR inhibitors, such as lipid and protein components [26,27].

Regarding direct detection in analyzing wound samples LAMP detected mecA gene in one sample (no.5), this sample was excluded as non mannitol fermenting staphylococcus and it may be $S$. epidermidis that is carrier for mecA gene[3,7,24,28] This sample was not detected by PCR. The direct detection results also support LAMP sensitivity over the PCR. However, both LAMP and PCR use in directly extracted samples failed to detect mecA gene in six (85.7\%) out of the total seven samples that were MRSA according to culture method (six wound samples and one dental plaque sample). The reason might be that directly extracted samples using boiling method produced insufficient amounts of DNA. LAMP and PCR perform better when using DNA extraction kit in direct detection of MRSA from dental plaque samples [7]. In direct analysis of the urine samples, the LAMP and PCR demonstrated identical results, suggesting the clinical applicability of the method for urine samples, However, this sample (one positive sample detected by LAMP and PCR) was excluded as coagulase negative Staphylococcus. 


\section{Conclusion}

Identification of MRSA and resistance of mec A gene is necessary for the surveillance of their transmission in hospitals and to overcome the problems associated with Gram+ve MRSA resistance. The LAMP described here is a reliable and rapid method for detection of the mec A gene in cultured organisms obviating the need for using antibiotics sensitivity testing.

Generally, these findings. should help faster and better management and treatment of the affected patients, in addition to rapid initiation of infection control procedures in health facilities. The results should also encourage future country wide epidemiological studies since there is little available information about MRSA infection in Sudan.

\section{Bibliography}

1. Khairalla AS., et al. "Carriage Frequency, Phenotypic, and Genotypic Characteristics of Methicillin-Resistant Staphylococcus aureus Isolated from Dental Health-Care Personnel, Patients, and Environment". Scientific Reports 7.1 (2017):1-16.

2. Ryan KJ and Ray CG "Sherris Medical Microbiology. An introduction to infectious diseases, New York: Mcgraw-Hill (2004).

3. Cimolai N. "MRSA and the environment". European Journal of Clinical Microbiology and Infectious Diseases 27 (2008): 481493.

4. Maina EK., et al. "Characterization of methicillin-resistant Staphylococcus aureus from skin and soft tissue infections in patients in Nairobi, Kenya". International Society for Infectious Diseases (2013): 115-119.

5. Dora N. "MRSA A Global Threat". MD Thesis Ut Southwestern Medical School (2011).

6. Earls MR., et al. "The Recent Emergence in Hospitals of Multidrug-Resistant Community-Associated Sequence Type 1 and Spa Type t127 Methicillin resistant Staphylococcus aureus Investigated by Whole-Genome Sequencing: Implications for Screening". Plos One 12.4 (2017): 1-17.

7. Koide Y., et al. "Rapid Detection of mec A and Spa by the LoopMediated Isothermal Amplification (LAMP) Method". Letters in Applied Microbiology 50.4 (2010): 386-392.
8. Su J., et al. "Rapid and Simple Detection of Methicillin-Resistant Staphylococcus aureus by orf X Loop-Mediated Isothermal Amplification Assay". BMC Biotechnology 14.1 (2014): 8.

9. Brown D. "Detection of Methicillin / Oxacillin Resistance in Staphylococci”. Journal of Antimicrobial Chemotherapy 48.1 (2001): 65-70.

10. Sudhaharan S., et al. "Evaluation of LAMP Assay Using Phenotypic Tests and Conventional PCR for Detection of Nuc and mecA Genes among Clinical Isolates of Staphylococcus SPP". Journal of Clinical and Diagnostic Research 9.8 (2015): DC06DC09.

11. Saadoun I., et al. "Frequency of MecA Gene and Susceptibility Pattern in Airborne Methicillin Resistant Staphylococcus aureus Isolated from Different Hospitals in North of Jordan". Sebha Medical Journal 12.2 (2013): 65-72.

12. Hiramatsu K., et al. "Analysis of border-line-resistant strains of methicillin-resistant Staphylococcus aureus using polymerase chain reaction". Medical Microbiology and Immunology 36 (1992): 445-453.

13. Makgotlho E., et al. "Molecular identification and genotyping of MRSA isolates". FEMS Immunology and Medical Microbiology 57 (2009): 104-115.

14. Notomi T., et al. "Loop-Mediated Isothermal Amplification of DNA". Nucleic Acids Research 28.12 (2000): e63.

15. Rittié L and Perbal B. "Enzymes Used in Molecular Biology: A Useful Guide". Journal of Cell Communication and Signaling 2.12 (2008): 25-45.

16. Uemura N., et al. "Development of a loop-mediated isothermal amplification method for diagnosing Pneumocystis pneumonia”. Journal of Medical Microbiology 57.1 (2008): 50-57.

17. Njiru ZK., et al. "Loop-mediated isothermal amplification (LAMP) method for rapid detection of Trypanosoma brucei rhodesiense". PLOS Neglected Tropical Diseases 2.2 (2008): e147.

18. Wang DG., et al. "Two methods for increased specificity and sensitivity in loop-mediated isothermal amplification". Molecules 20.4 (2015): 6048-6059.

19. Kalvatchev Z., et al. "Loop-mediated amplification for sensitive and specific detection of viruses". Biotechnology and Biotechnological Equipment 24.1 (2010):1559-1561. 
20. Lim KT., et al. "Loop-Mediated Isothermal Amplification Assay for the rapid Detection of Staphylococcus aureus". Bio Med Research International 895.816 (2013): 5.

21. Tie Z., et al. "Loop-Mediated Isothermal Amplification for Detection of Staphylococcus aureus in Dairy Cow Suffering from Mastitis". Journal of Biomedicine and Biotechnology 435.982 (2012): 1-5.

22. Liu Y., et al. "PCR-based Approaches for the Detection of Clinical Methicillin-resistant". The Open Microbiology Journal 10.12 (2016): 45-56.

23. Enomoto Y., et al. "Rapid Diagnosis of Herpes Simplex Virus Infection by a Loop-Mediated Isothermal Amplification Method". Journal of Clinical Microbiology 43.2 (2005): 951-955.

24. Savan R., et al. "Sensitive and Rapid Detection of Edwardsiellosis in Fish by a Loop-Mediated Isothermal Amplification Method". Applied and Environmental Microbiology 70.1 (2004): 621-624.

25. Okuda M., et al. "Characterization of the tufB-secE-nusG-rplKAJL-rpoB Gene Cluster of the Citrus Greening Organism and Detection by Loop-Mediated Isothermal Amplification". Plant Disease 89.7 (2005): 705-711.

26. Karanis P., et al. "Development and Preliminary Evaluation of a Loop-Mediated Isothermal Amplification Procedure for Sensitive Detection of Cryptosporidium Oocysts in Fecal and Water Samples". Applied and Environmental Microbiology 73.17 (2007): 5660-5662.

27. Kaneko H., et al. "Tolerance of loop-mediated isothermal amplifi- cation to a culture medium and biological substances". Journal of Biochemical and Biophysical Methods 70 (2007): 499-501.

28. Petinaki E., et al. "Detection of mecA, mecR1 and mecI Genes among Clinical Isolates of Methicillin-Resistant Staphylococci by Combined Polymerase Chain Reactions". Journal of Antimicrobial Chemotherapy 47 (2001): 297-304.

Volume 2 Issue 11 November 2019

(C) All rights are reserved by Khalid A Enan., et al. 